\title{
Exploring Natural Product Fragments for Drug and Probe Discovery
}

\author{
Axel Pahla , Herbert Waldmann ${ }^{\star a b}$, and Kamal Kumarª
}

\begin{abstract}
Fragment-based ligand discovery is a key technology to develop lead structures for drug discovery. The majority of the fragments employed so far is aromatic and $\mathrm{sp}^{2}$-configured, and there is a high demand of fragments with stereogenic centers. Natural products (NPs) are evolutionary selected ligands for a range of diverse macromolecular targets. Small-sized molecules - fragments - based on NPs may inherit the biological relevance of nature's treasure and could offer novel opportunities to engage challenging protein targets. An overview of this emerging research area is presented. The deconstruction of a complex NP into small fragments marks the beginning of this journey that is facilitated by the synthesis of NP-based 3D fragments. The emerging strategies in organic synthesis for either degradation of NPs to access fragments or de novo construction of fragments and their further combinations to chart novel biologically relevant chemical space is discussed.
\end{abstract}

Keywords: 3D Fragments · Fragment-based drug discovery $\cdot$ Natural product-fragments $\cdot$ Scaffolds $\cdot$ SCONP

\section{Introduction}

The quality of chemical leads, in particular their molecular properties influence the attrition rates in the search of new drugs. Often developmental drug candidates are subject to gradual decrease in molecular weight and lipophilicity as they proceed through each of the different clinical stages to become approved drugs. This observation led to the insight to employ low molecular weight compounds - fragments - in screening campaigns. The use of small fragments in drug discovery (FBDD) has now grown into a well-established technology. ${ }^{[1]}$ The central dogma of FBDD rests on the basis that by limiting molecular size, a relatively small number of fragments can cover a large fraction of accessible chemical space..$^{[2]}$ Thus, small molecular entities of limited size ( 150 to $300 \mathrm{~g} / \mathrm{mol})$ are tested for their binding to the target of in-

${ }^{*}$ Correspondence: Prof. Dr. H. Waldmann ${ }^{a b}$ Dr. K. Kumara

aMax Planck Institute of Molecular Physiology Department of Chemical Biology

Otto-Hahn- Str. 11, 44227-Dortmund, Germany

bFaculty of Chemistry and Chemical Biology

Technical University Dortmund

Otto-Hahn Str. 6, 44227-Dortmund, Germany

E-mail: herbert.waldmann@mpi-dortmund.mpg.de;

kamal.kumar@mpi-dortmund.mpg.de terest mostly by biophysical analysis (e.g. by surface plasmone resonance, NMR, or thermal shift). Hits from fragment libraries typically display weak affinities in the 100 $\mu \mathrm{M}$ to $10 \mathrm{mM}$ range, and thus the screening methodology must provide 100-1000-fold higher sensitivity. Thus, fragment screening routinely utilizes more sensitive biophysical technologies. Low affinity fragments are developed into new compounds with high affinity and drug-like properties either by growth of a hit fragment or, less frequently, by combination of fragments that bind to adjacent regions in the protein binding site. ${ }^{[3]}$ Compared to standard screening efforts, the fragment-based approach requires fewer compounds to be initially screened and can lead to a more efficient optimization process. The FBDD approach essentially represents a structurebased design method and is, therefore, mostly limited to target-based screening where the biological target can be isolated in sufficient amounts for the required X-ray and NMR studies. However, very recently some exciting reports of fragment-based phenotypic screenings and its application in target identification have emerged and appear to hold great promise in revealing novel targets for drug discovery research. ${ }^{[4]}$

In addition to the suitability of the target, the success rate of a fragment screening campaign largely depends on the quality and diversity of the fragment library. Most fragment libraries are traditionally derived from known drugs and lead compounds and therefore mainly cover an already explored chemical space. ${ }^{[5]}$ Many libraries are also overpopulated with flat and aromatic structures and lack diversity in their 3D shape, which further reduces the range of covered chemical space. ${ }^{[6]}$
Notably, the problem of 'flat' molecules has been associated with high attrition rates in drug discovery, due to their low solubility, promiscuity and Cyp450 inhibition. Consequently, an increase in stereogenic character and reduction of the number of aromatic rings is observed for development compounds during the drug discovery process. ${ }^{[7]}$ A large diversity in 3D shape is also a prerequisite for broad bioactivity. ${ }^{[8]}$ For these reasons, fragment libraries with a high diversity in structural classes and 3D shape are gaining attention and remain in high demand. ${ }^{[9]}$

NPs have been subject to the evolutionary process of screenings in their host organisms and represent the biologically chosen ligands for interaction with biological targets. Still a major share of successful drug discovery employs NP-based and derived small molecules. ${ }^{[10]}$ From penicillin antibiotics where the $\beta$-lactam ring ${ }^{[1]}$ plays a key role to attack the target proteins in bacteria to anti-diabetic lupins whose activity can be attributed to a quinolizidine ring, ${ }^{[12]}$ the structural design of these biosynthetic products complements the protein drug targets and therefore results in desired biological activities. In general but in contrast to combinatorial screening collections, NPs display a high number of stereogenic centers and diverse 3D geometry, as well as a low number of aromatic rings, low nitrogen count and a high number of oxygen atoms. ${ }^{[13]}$ NPs are biologically validated small molecules that cover the areas of chemical space typically not covered by synthetic small molecules. While the total synthesis of either the complex NPs or their truncated versions remain a practical challenge to establish compound collections, ${ }^{[14]}$ exploration of the fragments of 
NPs will provide novel opportunities for fragment-based approaches to find novel and first in class drugs. Given their interesting and unique structural features as compared to molecules of a usual screening collection, NPs are excellent starting points for the generation of fragments with desirable properties. ${ }^{[15]}$ Synthetic access to suitably functionalized NP fragments would provide novel opportunities to synthesize NP inspired compound collections and offer novel small molecules for drug discovery research. ${ }^{[3]}$

\section{The Deconstruction of Natural Products}

\subsection{Fragment Analysis}

FBDD relies on the weaker but quality interactions of fragments with the binding site of a protein and in general a large increase in the complexity of the molecules reduces the chances of a good match with the binding partner. Therefore, highly complex small molecules are not very suitable for FBDD. [16] Structural diversity nevertheless is highly important for any fragment-based library. A balance in the molecular properties is desirable to obtain the best results in FBDD. Pascolutti et al. analyzed the Dictionary of NPs (DNP) for NPs of fragment size and showed by analysis of physicochemical compound descriptors, pharmacophore features and structural diversity, that fragment-sized NPs retain to a large extent the pharmacophore features of larger NPs and that the molecules based on 2-ring scaffolds offer the best balance between minimal complexity and structural diversity. ${ }^{[15 a]}$

Several approaches to deconstruct NPs (and organic molecules in general) into suitable fragments have been described.

Starting from the NP, as a first step the scaffold has to be identified. This is generally done based on the structure by using the procedure defined in the seminal paper by Bemis and Murcko. ${ }^{[17]}$ In short, a scaf- fold is generated from the parent molecule by removal of terminal chains and ring substituents. The scaffold is then gradually deconstructed into smaller fragments. One approach to do this is by removing rings and bonds successively following a set of prioritization rules. This ensures that a given scaffold is deconstructed linearly and on each step of the deconstruction only one fragment is generated.

This approach underlies the structural classification of NPS (SCONP) and was originally used to chart the biologically relevant chemical space by classifying NPs ${ }^{[18]}$ and led to the definition of the Scaffold Tree, which employs prioritization rules that aim to identify chemically intuitive scaffolds. ${ }^{[19]}$ The major feature of the scaffold tree approach is its linearity. Independent of the size of the scaffold, the deconstruction only yields one fragment at each step, avoiding a fragment 'explosion' and allowing easy visualization (Fig. 1). Depending on the set of prioritization rules used and the task at hand, this might also be a disadvantage, as meaningful fragments might be overlooked. With the program Scaffold Hunter an open-source tool for generating and visualizing scaffold trees is available. [20]

Another approach to scaffold fragmentation is scaffold network. Originally described as Hierarchical Scaffold Clustering (HierS), ${ }^{[21]}$ the scaffold network consists of all possible combinations that can be generated by pruning rings from the parent scaffold, generating a scaffold pyramid. Due to the larger number of generated scaffolds, the network covers scaffolds which are not generated in the scaffold tree approach. This was shown to be advantageous in the analysis of compound set enrichment, where statistically enriched fragments and scaffolds were identified by mapping biological activity to the scaffold substructure relationships generated by the scaffold network. ${ }^{[22]}$

Other methods for the generation of scaffolds and fragments focus on different

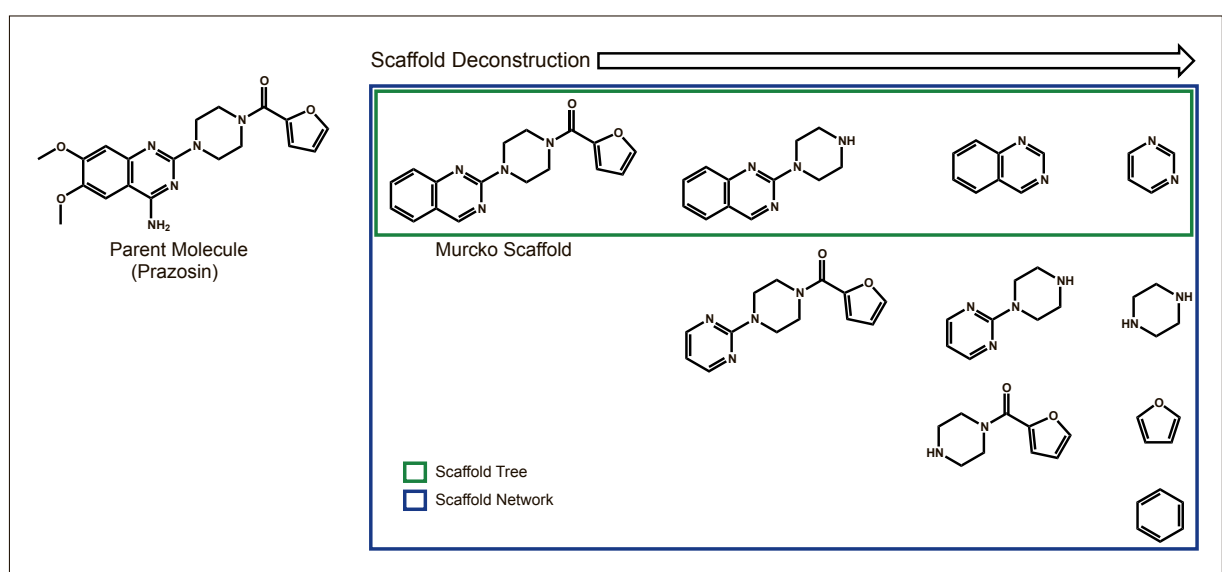

Fig. 1. Scaffold tree approach to deconstruct NPs.

aspects, e.g. the RECAP and the SynDiR methods use retrosynthetic aspects ${ }^{[23]}$ and the recently published ASB method considers compound analogue series. ${ }^{[24]}$

\subsection{NP Deconstruction to Yield Novel Bioactive Fragments}

The most straight-forward procedure to generate NP fragments is to deconstruct the known NPs (e.g. from the DNP) by one of the above described methods. This has, for example, been done in the SCONP study and by Over et al. ${ }^{[25]}$ using a modified scaffold tree procedure that retained both the attachment points and the directly attached functional groups and side chains were shortened for later fragment linking and growing. Also, carbonyl groups were treated as single heteroatoms. Importantly, to conserve greater $s p^{3}$ content in the fragments, the hybridization and configuration of the centers were not changed during the deconstruction of fused ring-systems as depicted in Fig. 2 for the deconstruction of the NP renieramycin $P$.

Fragmentation analysis of 183,769 NP structures from DNP 18.2 afforded 751,577 NP-fragments. Further filtering to remove the potentially toxic, unstable or highly reactive fragments and followed by retaining fragments following criteria close to the 'rule of three' $(\mathrm{A} \log \mathrm{P}<3.5$, a molecular weight of 120-350 Da, $<3$ hydrogen bond donors, $\leq 6$ hydrogen bond acceptors) yielded around 160,000 fragments. Further removal of molecules that were macrocycles, had multi-bridged structures, as well as using iterative clustering to remove structurally similar scaffolds resulted in 2000 clusters of fragments with structural diversity and NP properties (Fig. 3).

Around 190 cluster centers or closely related stable analogues were either procured from commercial vendors or synthesized and they also delivered unexplored ligand classes of chemical space for the established drug targets p38 $\alpha$ MAP kinase. For instance, fragment 1 of a 85 -member cluster represent 53 NPs such as sparteine (2). Experimentally, $\mathbf{1}$ and $\mathbf{2}$ were identified as weak inhibitors of p38 $\alpha$ MAP kinase. Further synthesis of the cytisine and sparteine derivatives and the subsequent co-crystal structures with the protein revealed that $\mathbf{5}$ and $\mathbf{6}$ bind to the allosteric pocket of $\mathrm{p} 38 \alpha$ MAP kinase as novel class of type III inhibitors (Fig. 4).

The unique binding characteristics of these NP-fragments resulting in allosteric kinase inhibition is of great interest in modern kinase inhibitor research.[26] Compounds 5 and $\mathbf{6}$ represent novel type III inhibitors, which bind exclusively to the allosteric site and explore less conserved sites to increase kinase inhibitor selectivity. ${ }^{[27]}$ Thus, the unique molecular shape of $s p^{3}$-rich fragments 5 and $\mathbf{6}$ offers unprec- 


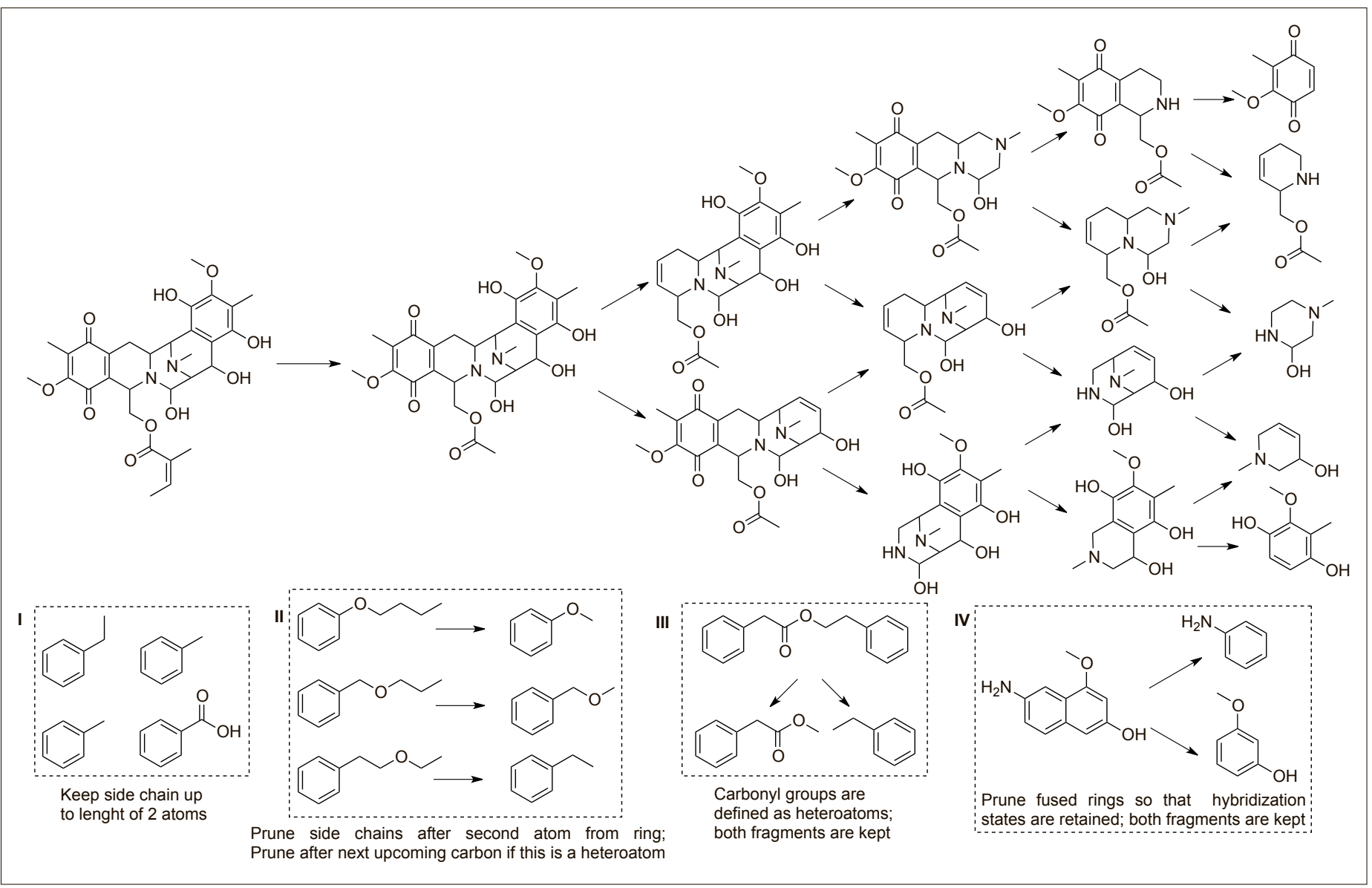

Fig. 2. Generation of fragments from NP renieramycin P. (from Over et al., Nat Chem, 2013, 5, 21-28; DOI: 10.1038/NCHEM.1506).[25]

edented starting points for further kinase inhibitor research.

\subsection{Natural Product-likeness of Fragments}

Although a scaffold or fragment found by this approach may by definition be called a NP fragment, this does not necessarily mean that it has the desired properties of a high fraction of $s p^{3}$ content or 3D shape that usually are associated with NPs and practically render a fragment NP-like. Interestingly, saturation i.e. $s p^{3}$ content as well as the presence of stereocenters increase and the aromatic content decreases in the transition from early discovery to drugs through clinical trials. Consequently, recent interest sharply increased in $s p^{3}$-rich and 3D-shaped libraries. ${ }^{[28]}$ In this context, it is probably more meaningful to discuss NP likeness of the screening fragments. Ertl et al. ${ }^{[29]}$ introduced the NP-likeness score as a similarity measure of a molecule to the chemical space occupied by NPs in general. This method is widely applicable because it does not require the minimized 3D structure of the molecules. An implementation of the algorithm can be $e . g$. found in the open-source cheminformatics toolkit RDKit (http://www.rdkit. org). While the method was designed for classifying compounds it can also be used for prioritizing fragments. ${ }^{[9]}$

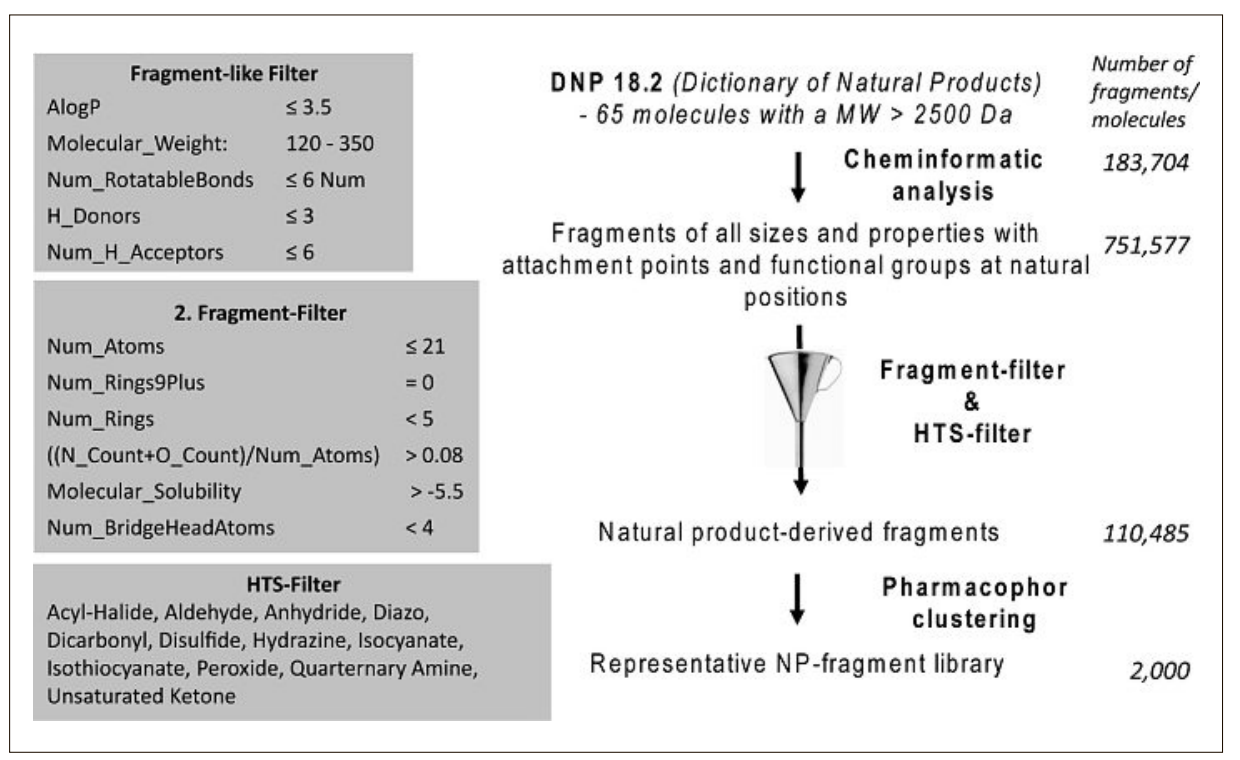

Fig. 3. Filtering and clustering of NP fragments (from Over et al., Nat Chem, 2013, 5, 21-28; DOI: 10.1038/NCHEM.1506). ${ }^{[25]}$

The NP likeness score takes multiple properties of NPs into consideration. For instance, the fraction of $s p^{3}$ carbons $\left(\mathrm{Fsp}^{3}\right)$, originally described by Lovering et al. to characterize development compounds, ${ }^{[7 \mathrm{~b}]}$ is high in NPs and can be used to classify fragments for their NP similarity. It is very easy to calculate and again does not require the 3D structure of the molecule.
Another aspect of NPs, their 3D shape, can be calculated using different methods, e.g. Principal Moment of Inertia (PMI) ${ }^{[8]}$ and Plane of Best Fit (PBF). ${ }^{[30]}$ PMI were evaluated by Sauer et al. to describe molecular 3D shapes. These can be visualized as the typical triangle-shaped plots of normalized PMI ratios (NPR), where the rod, disc and sphere (represented by acety- 


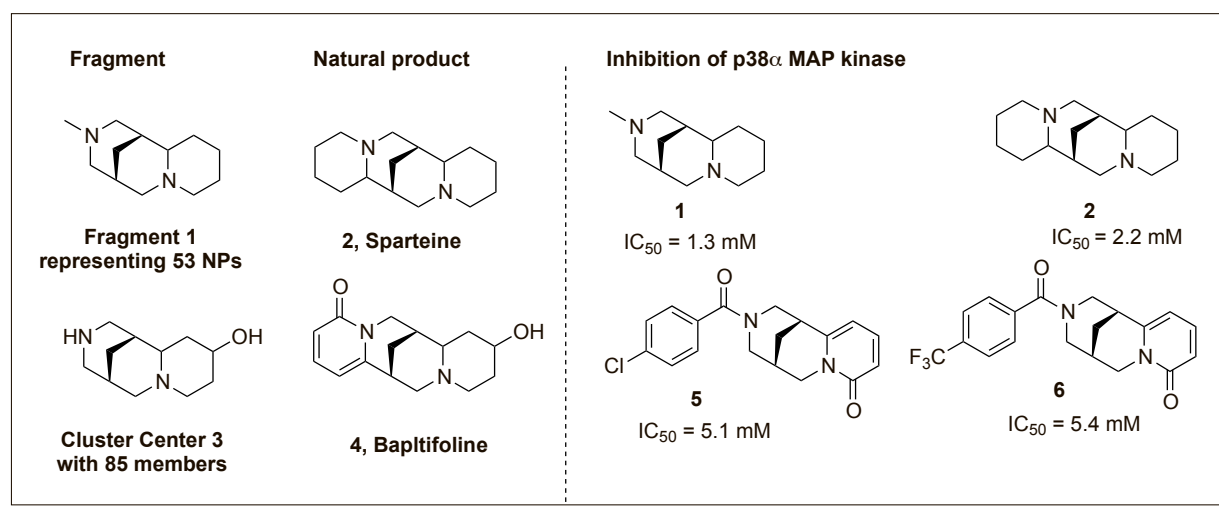

Fig. 4. NP-derived fragment and their derivatives as kinase inhibitors.

lene, benzene and adamantane) are at the corners of the triangle (Fig. 5). PBF was described by Firth et al. ${ }^{[30]}$ as a quantitative measure of how far away the atoms in the minimized 3D structure of a molecule are from the plane of best fit through the molecule.

\section{Synthesis Efforts to Generate NP-Fragments}

Libraries of NP fragments significantly broaden the accessible chemical space compared to traditional fragment libraries. Prescher et al. showed that a library of NP-like fragments enlarged the chemical space in comparison to an in-house fragment library. ${ }^{[9]}$ In recent years, considerable efforts have been taken by the synthesis community to address the limitations and drawbacks of commercial fragment libraries, in particular the lack of 3D structural features, like stereocenters and the NP-likeness of fragments. Different synthesis approaches have been followed to access NP-derived and inspired fragments. In general, three categories of synthesis efforts are followed to produce NP-like fragments (Fig. 6): i) degradation of large NPs with or without in silico guidance,

ii) chemical modifications of NPs into suitable fragments and

iii) exploring commercial or synthetic fragments for the synthesis of novel NP-like scaffolds

\subsection{Degradation of Large NPs}

The often inefficient synthetic tractability of highly complex NPs remains the most important factor that disfavors NPs in drug discovery. Such strategies have already been discussed in other reviews and are not covered in this review article. On the other hand, the availability of complex NPs can be exploited to construct low molecular weight smaller fragments. Identification of any small molecule based on these fragments exhibiting interesting biological activity would further call for developing efficient synthesis towards these NP-fragments. Furthermore, molecular rearrangements of complex NPs also pave the way to novel NP-analogues.

The fragmentation approach to degrade a NP can be illustrated with the example of sanglifehrin A. ${ }^{[32]}$ A multistep cascade starting with known dihydroxylation and periodate cleavage of the NP yielded fragment 7. A sequence of further eight steps delivered the desired spiroaminal fragment $\mathbf{8}$ that could be used to synthesize a collection of NP-fragment derivatives (Scheme 1).

An additional example that provided novel, unprecedented NP chemistry of interest is the degradation of cytochalasin $\mathrm{E}^{[33]}$ (Scheme 2). Ozonolysis under two different conditions resulted in two aldehydes with and without an epoxide 9 and 10 respectively. Whereas the reduction of intermediate aldehyde 9 yielded novel fragment 14, non-reductive workup of the ozonolysis reaction with pyridine provided the novel aldehyde 10. Aldehyde $\mathbf{1 0}$ was further transformed into novel fragment 11 by reductive amination and fragments 12 and 13 by oxidation or reduction, respectively.

\subsection{Chemical Modification of NPs}

In contrast to the deconstruction of high molecular weight NPs, a number of fragments can also be obtained by derivatization or modification of small NPs. Hergenrother and co-workers had developed a ring-distortion synthesis approach wherein core-ring structures of readily available and structurally complex NPs were selectively rearranged in few chemical steps into markedly diverse and distinct core scaffolds.[34] This strategy can be explored to provide modified fragments of available NPs. For instance, gibberellic acid (Scheme 3), that is produced industrially on the ton scale is a plant hormone isolated from Gibberella fujikuroi. On its tetracyclic diterpene core, gibberellic acid contains a fused lactone, two allylic alcohols, an exocyclic olefin and a carboxylic acid, which offer opportunities for the selective functionalization of each ring of the tetracyclic core. Towards a smaller NP fragment, treatment of gibberellic acid with dilute hydrochloric acid induced the elimination of the lactone and decarboxylation

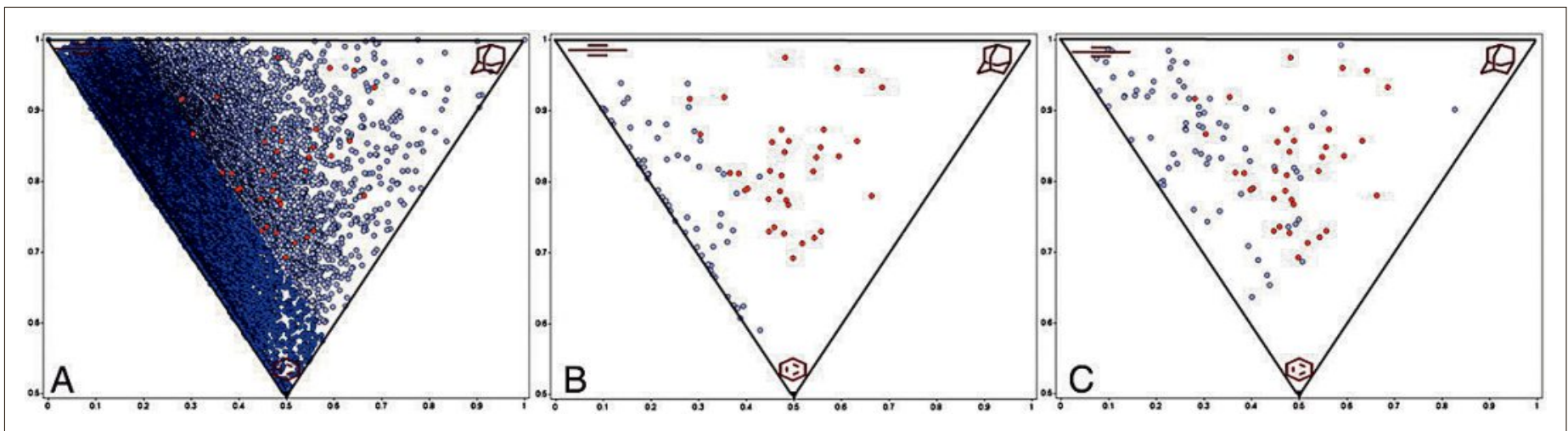

Fig. 5. Principal Moments of Inertia Plots. (A) PMI plot depicting 35 3D fragments and 18,534 fragments obtained from ZINC database (gray). The darker-blue region represents $75 \%$ of the 18,534 fragments. (B) PMI plot depicting 35 3D fragments and 62 best-matched fragments from ZINC (gray), based on heavy atom count similarity. (C) PMI plot depicting 35 3D fragments and 76 of best-matched fragments from ZINC based on physiochemical descriptors. Reproduced with permission from Hung et al., Proc. Natl. Acad. Sci. 2011, 108, 6799-6804; doi: 10.1073/pnas.1015271108). ${ }^{[1]}$ 
Fig. 6. Synthesis strategies to generate NP-fragments.
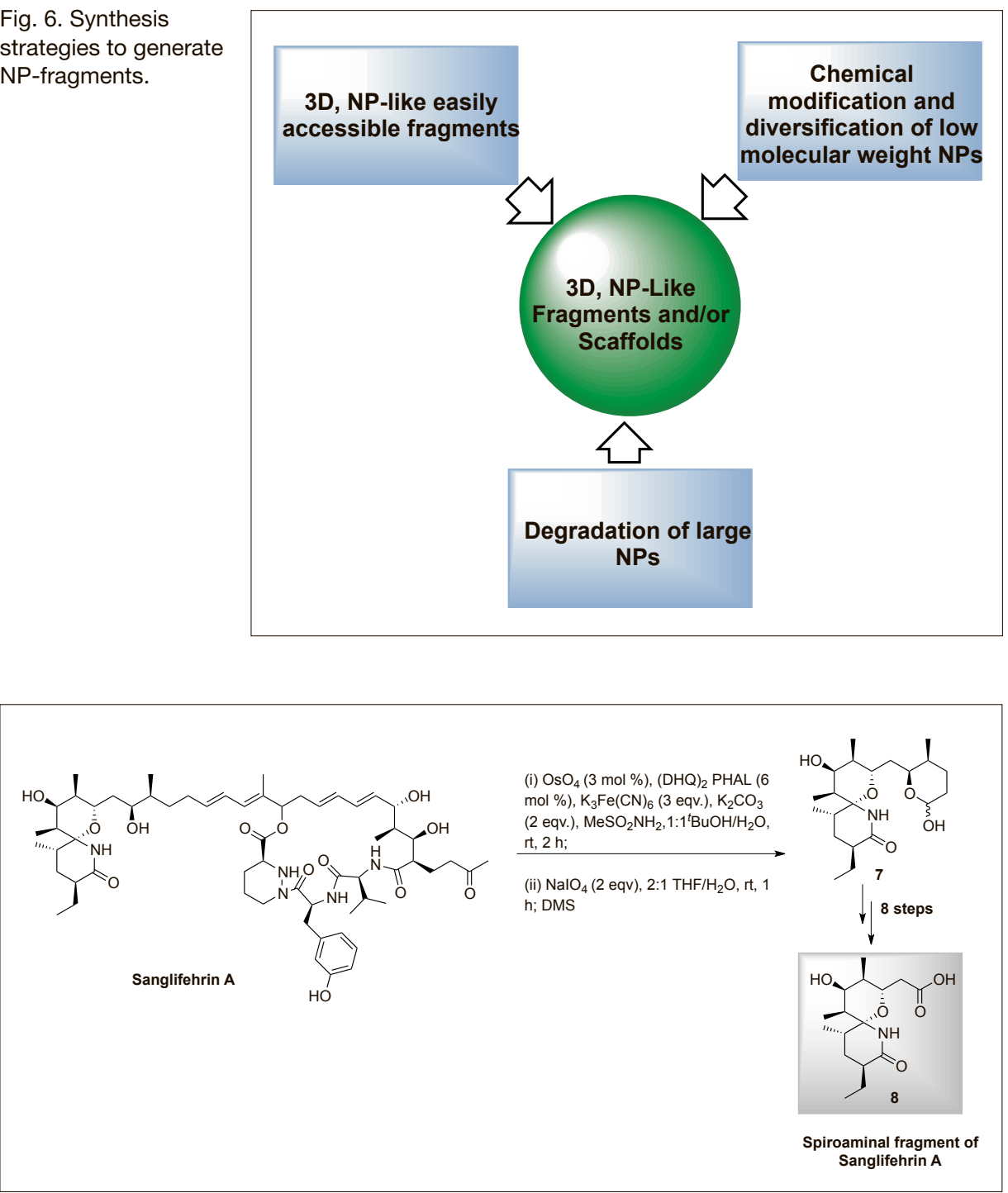

Scheme 1. Fragmentation of sanglifehrin $A$.

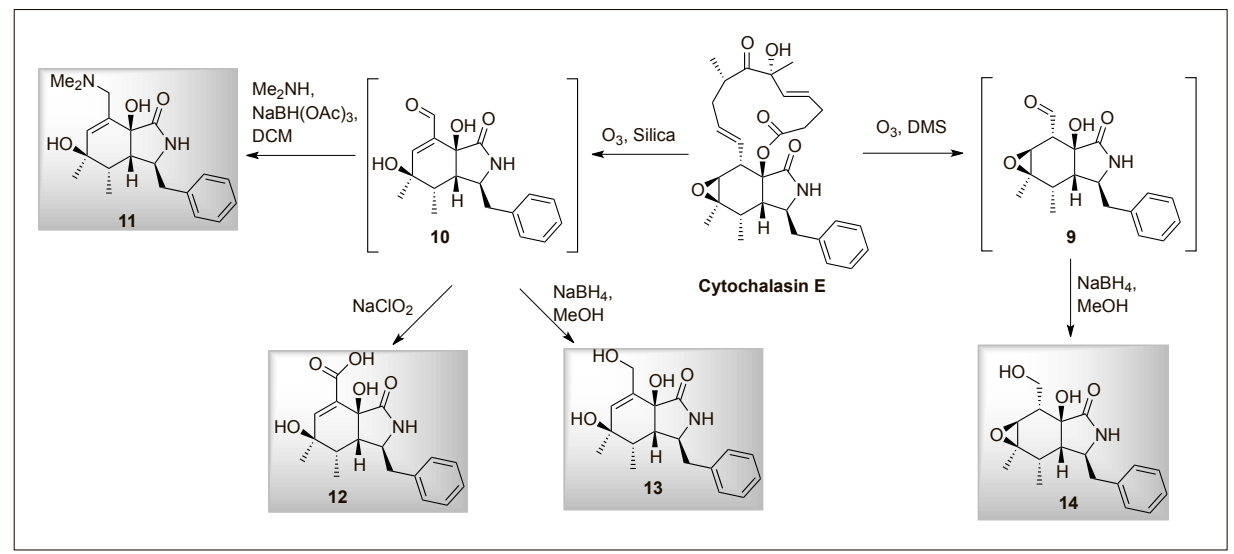

Scheme 2. Fragment generation by degradation of cytochalasin E.

to aromatize the A ring, which enables the isolation of allo-gibberic acid (15). On the other hand, in refluxing hydrochloric acid gibberellic acid underwent aromatization and a Wagner-Meerwein rearrangement to form fragment $\mathbf{1 6}$ (Scheme 3). Both fragments had functional groups available for the synthesis of additional small molecules.
19 was isolated as side product of the prolonged treatment of the NP massarigenin $\mathrm{C}$ with $\mathrm{HCl}$. This interesting fragment was further derivatized using the carboxylic acid as handle in reduction and amide synthesis reactions.

Diversifying a NP including its truncation needs to exploit the available modifiable functional groups. The structurally complex steroidal framework of the NP adrenosterone A (Scheme 5) contains five contiguous stereogenic centers. Also, each of the four individual carbocyclic rings of $\mathrm{A}$ is functionalized with an enone or ketone. While the A ring has an enone that is connected to the B ring by an exocyclic double bond, ring $C$ and $D$ are each functionalized with a ketone. During these synthetic investigations of androsterone by the Hergenrother group, a novel substrate dependent Schmidt reaction was discovered that afforded both ring expansion and ring cleavage in a single synthetic transformation to yield fragment 20. A tandem D-ring cleavage and A-ring expansion under Schmidt conditions for one hour afforded $\mathbf{2 0}$ as the major product (along with its constitutional isomer). Reduction of the newly generated enone afforded the tertiary alcohol and removed the reactive Michael acceptor function in the NP fragment (Scheme 5).Treatment of the alcohol intermediate with acetic anhydride in pyridine with catalytic 4-dimethylaminopyridine (DMAP) affords 21 as NP-fragment.

\subsection{Modulating NP-Fragments}

Compound classes that are derived from or inspired by NPs represent chemical space explored by nature in evolution and thus could offer biologically prevalidated starting points for the development of bioactive compounds. In recent years, NP-inspired compounds have successfully led many chemical-biological investigations of cellular signaling cascades. Traditionally FBDD selects mildly active fragments and based on their interaction with the protein targets, growth of the fragments is further designed. However, considering the privileged structures of many NP fragments and the fact that organic chemistry has been overtly conserved in its exploration of NP chemical space, ${ }^{[35]}$ approaches that afford novel small-sized NP-scaffolds could provide interesting bioactive molecules for probe and drug discovery research. A major advantage in this case is the non-dependency on either NPs or their truncated parts and the possibility to scale-up the synthesis to get a real small molecule library for different screening campaigns.

As a representative example, the Waldmann group developed a synthesis strategy to merge interesting NP fragments 
Scheme 3.

Fragmentation of complex gibberellic acid.

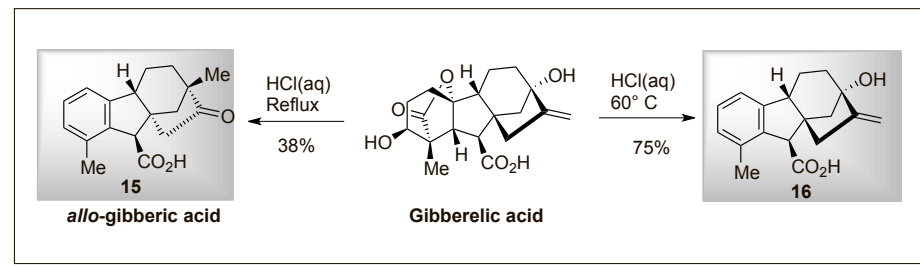

Scheme 4 Modification of massarigenin $C$ to provide NP-fragments.

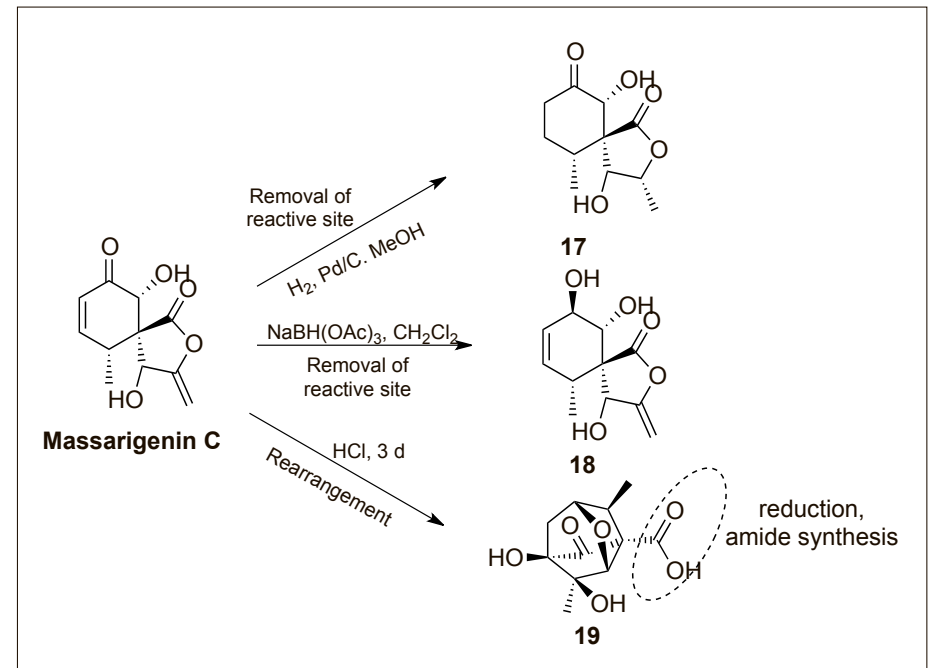

different kinases.[40] The neuroenhancing pyridones were profiled for the inhibition of 67 kinases involved in neurite outgrowth and both compounds inhibited the kinase MAP4K4 (mitogen-activated protein kinase 4, also termed HPK/GCK-like kinase HGK or MEKKK4) with $\mathrm{IC}_{50}$ values in the range of 1.9-3.6 $\mu \mathrm{M}$. The potency of MAP4K4 inhibition directly correlated with the degree of neuritogenic activity (Fig. 7b,c). The crystal structure revealed that 27a binds the kinase in a type I fashion (Fig. 7d). The pyridone ring of the molecule forms two hydrogen bonds to the kinase hinge region, thus engaging the peptide backbone of Glu106 and Cys108. The glycine-rich loop is folded over the inhibitor in a way that the side-chain of Tyr36 partly shields the 4-hydroxy-2-pyridone core of the NP-fragment from the solvent and consequently stabilizes the inhibitor binding (Fig. 7d).

These examples represent numerous unexplored opportunities wherein retaining desired effects of a NP in a minimized fragment could offer novel starting points for medicinal chemistry or chemical biology investigations.

\section{Conclusions}

Although FBDD is well-established in drug discovery with two approved drugs emanating from this approach, ${ }^{[3]}$ new ideas, methods and modalities are needed to advance this field of research to further successful applications. ${ }^{[41]}$ In particular, to tackle difficult or novel targets for which no chemical matter is found in existing HTS collections, fragments need to be explored from novel areas of chemical space, in particular, based on natural product structures. Biological relevance is the most important criterion to be met by compounds used in the discovery of protein ligands and inhibitors. ${ }^{[42]}$ Natural products perfectly meet this criterion as they are secondary metabolites selected by the evolutionary process. Fragments derived and based on natural product structures, by analogy, inherit the biological relevance and collectively represent the chemical space defined by the structures of natural products. Also, it is more economical to establish and screen a natural product-fragment library of a few hundred compounds than a standard HTS collection of e.g. $\geq 0.5$ million members. Different algorithms have been developed to dissect complex natural product structure to identify smaller fragments and /or ring-systems. Among the available programs, SCONP analysis, Scaffold HUNTER, HierS etc. can be easily used to deconstruct a natural product into its fragments. Smaller NP-fragments or building blocks often are not commercially availapproaches are very likely to offer novel of the HH-signaling pathway by tropanes or analogues thereof has not been observed before and thus this strategy unraveled a novel class of small molecule modulators of a very important signaling pathneurite outgrowth in primary neuron cells. Neuroprotection and neurite outgrowth have been linked to the modulation of

tool compounds to understand both the relthe starting points for medicinal chemisty nvestigations into novel structural classes. that decorates a plethora of biologically active pyridone alkaloids, like militarinone genic activity in PC-12 cells, was target by Waldmann and co-workers. Gademann simplified pyridone small molecule ing the side chain of militarinone D and demonstrated neuritogenic activity in a and co-workers synthesized a militarinone dimethoxypyridine (26, Fig. 7) that transformed into substituted pyridones (27) in four steps. ${ }^{[39]}$ The collection revealed a number of pyridones that induced 
able and therefore organic synthesis needs to play a pivotal role to offer efficient access to natural product fragment space. In particular, methods that seek to combine different fragments into novel scaffolds are gaining attention and call for further development. Combinations of fragments that include hybrids of fragments of a natural product and drug molecule ${ }^{[36]}$ could provide unique structural features and offer numerous opportunities to engage novel therapeutically relevant targets.

Received: June 22, 2017

[1] C. W. Murray, D. C. Rees, Nat. Chem. 2009, 1, 187.

[2] S. D. Bembenek, B. A. Tounge, C. H. Reynolds, Drug Discov. Today 2009, 14, 278.

[3] G. M. Keseru, D. A. Erlanson, G. G. Ferenczy, M. M. Hann, C. W. Murray, S. D. Pickett, J. Med. Chem. 2016, 59, 8189.

[4] C. G. Parker, A. Galmozzi, Y. J. Wang, B. E. Correia, K. Sasaki, C. M. Joslyn, A. S. Kim, C. L. Cavallaro, R. M. Lawrence, S. R. Johnson, I. Narvaiza, E. Saez, B. F. Cravatt, Cell 2017, 168, 527.

[5] a) K. Babaoglu, B. K. Shoichet, Nat. Chem. Biol. 2006, 2, 720; b) R. E. Hubbard, I. Chen, B. Davis, Curr. Opin. Drug Disc. 2007, 10, 289; c) M. G. Siegel, M. Vieth, Drug Discov. Today 2007, 12,71 .

[6] A. D. Morley, A. Pugliese, K. Birchall, J Bower, P. Brennan, N. Brown, T. Chapman, M Drysdale, I. H. Gilbert, S. Hoelder, A. Jordan, S. V. Ley, A. Merritt, D. Miller, M. E. Swarbrick, P. G. Wyatt, Drug Discov. Today 2013, 18, 1221.

[7] a) F. Lovering, Medchemcomm 2013, 4, 515; b) F. Lovering, J. Bikker, C. Humblet, J. Med. Chem. 2009, 52, 6752 .

[8] W. H. B. Sauer, M. K. Schwarz, J. Chem. Inf. Comp. Sci. 2003, 43, 987.

[9] H. Prescher, G. Koch, T. Schuhmann, P. Ertl, A. Bussenault, M. Glick, I. Dix, F. Petersen, D. E. Lizos, Bioorg. Med. Chem. 2017, 25, 921.

[10] a) M. Feher, J. M. Schmidt, J. Chem. Inf. Comp. Sci. 2003, 43, 218; b) T. Rodrigues, D. Reker, P. Schneider, G. Schneider, Nat. Chem. 2016, 8 , 531.

[11] K. F. Kong, L. Schneper, K. Mathee, Apmis 2010, 118,1 .

[12] B. Brunmair, Z. Lehner, K. Stadlbauer, I. Adorjan, K. Frobel, T. Scherer, A. Luger, L. Bauer, C. Furnsinn, Plos One 2015, 10, e0126847.

[13] a) S. Wetzel, A. Schuffenhauer, S. Roggo, P. Ertl, H. Waldmann, Chimia 2007, 61, 355; b) T. Henkel, R. M. Brunne, H. Muller, F. Reichel, Angew. Chem. Int. Ed. 1999, 38, 643.

[14] M. Garcia-Castro, S. Zimmermann, M. G. Sankar, K. Kumar, Angew. Chem. Int. Ed. 2016, 55,7586 .

[15] a) M. Pascolutti, M. Campitelli, B. Nguyen, N. Pham, A. D. Gorse, R. J. Quinn, Plos One 2015, 10, e0120942; b) E. A. Crane, K. Gademann, Angew. Chem. Int. Ed. 2016, 55 , 3882 .

[16] P. Selzer, H. M. Roth, P. Ertl, A. Schuffenhauer, Curr. Opin. Chem. Biol. 2005, 9, 310.

[17] G. W. Bemis, M. A. Murcko, J. Med. Chem. 1996, 39, 2887

[18] M. A. Koch, A. Schuffenhauer, M. Scheck, S. Wetzel, M. Casaulta, A. Odermatt, P. Ertl, H. Waldmann, Proc. Natl. Acad. Sci. USA 2005, 102, 17272 .

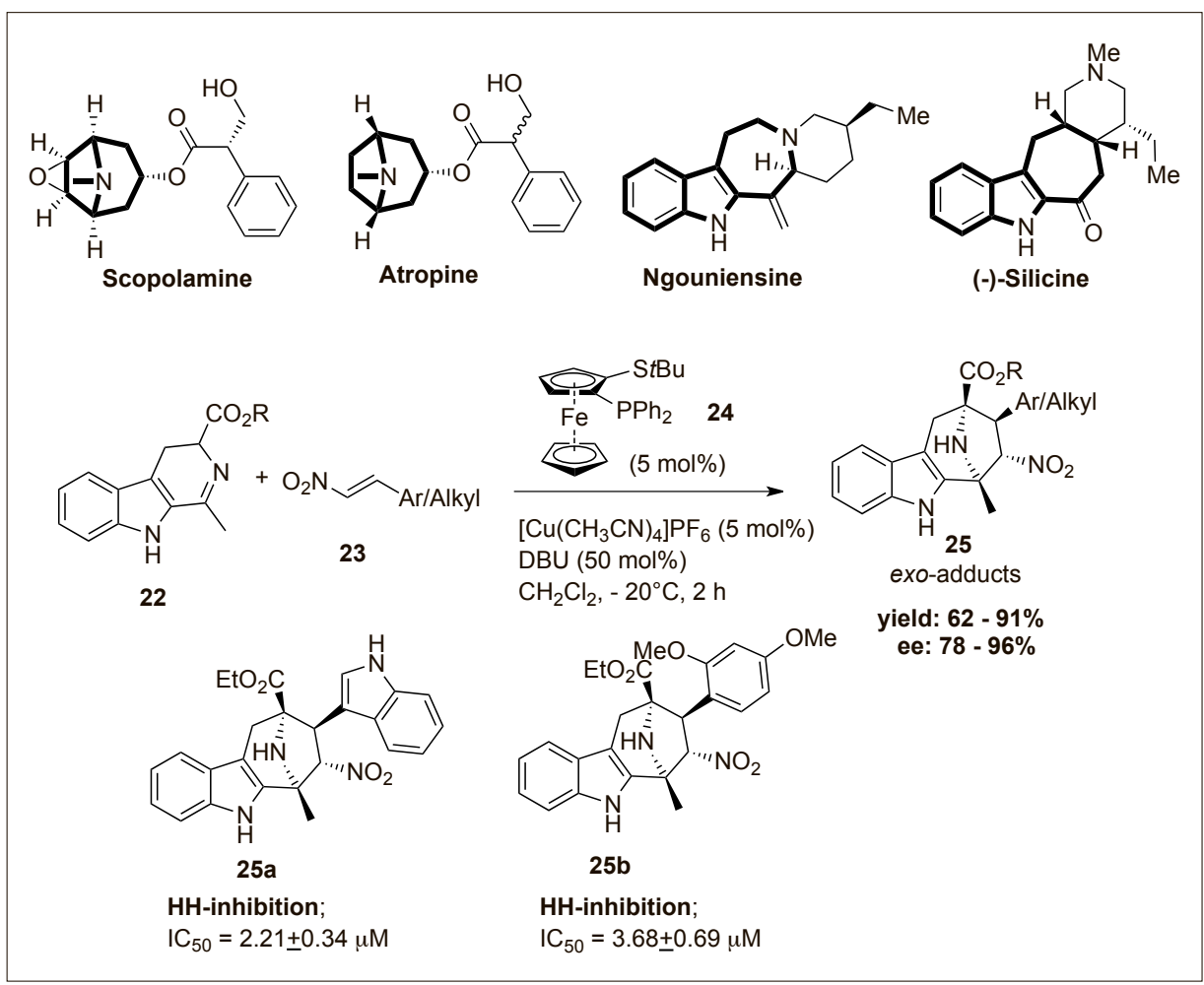

Scheme 6. Exploration of cycloaddition chemistry to combine NP-fragments.

a) $\mathrm{HO}$<smiles>CCC(C)CC(C)CC(C)C=CC=CC=CC(=O)C1C(=O)NCC(c2ccccc2)C1O</smiles><smiles>CCC(C)C=C(C)C=C(C)C=CC=CC(=O)c1c(O)c(-c2ccc(O)cc2)c[nH]c1=O</smiles>

Militarinone D

Farinosone A

b)<smiles>[R]C(=O)c1c(O)c([Al]CC#CC)c[nH]c1=O</smiles>

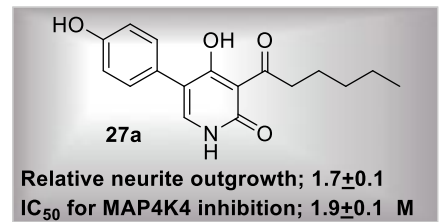

c)

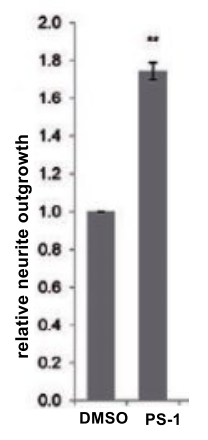

d)

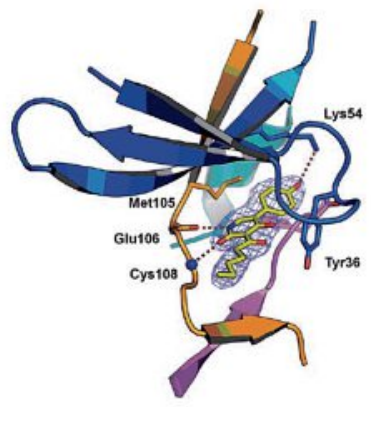

Fig. 7. a) NPs with pyridone scaffold; b) synthesis of substituted 4-hydroxy-pyridone derivatives (27); c) neurite outgrowth induced by 27 a pyridone molecule and d) The compound PS-1 complexed with wild-type MAP4K4.

[19] A. Schuffenhauer, P. Ertl, S. Roggo, S. Wetzel, M. A. Koch, H. Waldmann, J. Chem. Inf. Model 2007, $47,47$.

[20] S. Wetzel, K. Klein, S. Renner, D. Rauh, T. I. Oprea, P. Mutzel, H. Waldmann, Nat. Chem. Biol. 2009, 5, 581.

[21] S. J. Wilkens, J. Janes, A. I. Su, J. Med. Chem. 2005, 48,3182 .
[22] T. Varin, A. Schuffenhauer, P. Ertl, S. Renner, $J$. Chem. Inf. Model 2011, 51, 1528.

[23] a) X. Q. Lewell, D. B. Judd, S. P. Watson, M. M. Hann, J. Chem. Inf. Comp. Sci. 1998, 38, 511; b) N. C. Firth, B. Atrash, N. Brown, J. Blagg, J. Chem. Inf. Model 2015, 55, 1169.

[24] D. Dimova, D. Stumpfe, Y. Hu, J. Bajorath, Future Sci. 2016, 2, DOI: $10.4155 /$ fsoa-2016-0058 
[25] B. Over, S. Wetzel, C. Grutter, Y. Nakai, S. Renner, D. Rauh, H. Waldmann, Nat. Chem. 2013, 5, 21.

[26] M. Rabiller, M. Getlik, S. Kluter, A. Richters, S. Tuckmantel, J. R. Simard, D. Rauh, Arch. Pharm. 2010, 343, 193

[27] Y. M. Ahn, M. Clare, C. L. Ensinger, M. M Hood, J. W. Lord, W. P. Lu, D. F. Miller, W. C. Patt, B. D. Smith, L. Vogeti, M. D. Kaufman, P. A. Petillo, S. C. Wise, J. Abendroth, L. Chun, R. Clark, M. Feese, H. Kim, L. Stewart, D. L. Flynn, Bioorg. Med. Chem. Lett. 2010, 20, 5793.

[28] P. Ertl, A. Schuffenhauer, in 'Natural compounds as drugs, Volume II', Birkhäuser Verlag AG, Basel, Switzerland, 2008, p. 217.

[29] P. Ertl, S. Roggo, A. Schuffenhauer, J. Chem. Inf. Model 2008, 48, 68.

[30] N. C. Firth, N. Brown, J. Blagg, J. Chem. Inf. Model 2012, 52, 2516.

[31] A. W. Hung, A. Ramek, Y. K. Wang, T. Kaya, J. A. Wilson, P. A. Clemons, D. W. Young, Proc. Natl. Acad. Sci. USA 2011, 108, 6799.

[32] a) T. Fehr, J. Kallen, L. Oberer, J. J. Sanglier, W. Schilling, J. Antibiot. 1999, 52, 474; b) J. J. Sanglier, V. Quesniaux, T. Fehr, H. Hofmann,
M. Mahnke, K. Memmert, W. Schuler, G. Zenke, L. Gschwind, C. Maurer, W. Schilling, J. Antibiot. 1999, 52, 466.

[33] a) D. C. Aldridge, B. F. Burrows, W. B. Turner, J. Chem. Soc. Chem. Comm. 1972, 148; b) G. Buchi, Y. Kitaura, S. S. Yuan, H. E. Wright, J. Clardy, A. L. Demain, Glinsuko.T, N. Hunt, G. N. Wogan, J. Am. Chem. Soc. 1973, 95, 5423.

[34] R. W. Huigens, K. C. Morrison, R. W. Hicklin, T. A. Flood, M. F. Richter, P. J. Hergenrother, Nat. Chem. 2013, 5, 195.

[35] A. H. Lipkus, Q. Yuan, K. A. Lucas, S. A. Funk, W. F. Bartelt, R. J. Schenck, A. J. Trippe, J. Org. Chem. 2008, 73, 4443.

[36] R. D. Taylor, M. MacCoss, A. D. G. Lawson, J. Med. Chem. 2017, 60, 1638.

[37] K. Schmidt, W. Gunther, S. Stoyanova, B. Schubert, Z. Z. Li, M. Hamburger, Org. Lett. 2002, 4, 197.

[38] a) H. J. Jessen, A. Schumacher, T. Shaw, A. Pfaltz, K. Gademann, Angew. Chem. Int. Ed. 2011, 50, 4222; b) F. Schmid, H. J. Jessen, P. Burch, K. Gademann, Medchemcomm 2013, 4, 135.

[39] P. Schroder, T. Forster, S. Kleine, C. Becker, A. Richters, S. Ziegler, D. Rauh, K. Kumar,
H. Waldmann, Angew. Chem. Int. Ed. 2015, 54, 12398.

[40] a) S. Mehan, H. Meena, D. Sharma, R. Sankhla, J. Mol. Neurosci. 2011, 43, 376; b) S. H. Y. Loh, L. Francescut, P. Lingor, M. Bahr, P. Nicotera, Cell Death Differ. 2008, 15, 283; c) H. Al-Ali, S. C. Schurer, V. P. Lemmon, J. L. Bixby, ACS Chem. Biol. 2013, 8, 1027.

[41] L. Sancineto, S. Massari, N. Iraci, O. Tabarrini, Curr. Med. Chem. 2013, 20, 1355.

[42] a) S. Wetzel, R. S. Bon, K. Kumar, H. Waldmann, Angew. Chem. Int. Ed. 2011, 50, 10800; b) K. Kumar, H. Waldmann, Angew. Chem. Int. Ed. 2009, 48, 3224; c) S. Renner, W. A. L. van Otterlo, M. D. Seoane, S. Mocklinghoff, B Hofmann, S. Wetzel, A. Schuffenhauer, P. Ertl, T. I. Oprea, D. Steinhilber, L. Brunsveld, D. Rauh, H. Waldmann, Nat. Chem. Biol. 2009, 5 , 585 . 